\title{
Teleoperation with Kinematically Redundant Robot Manipulators with Sub-Task Objectives
}

\author{
Nitendra Nath^, Enver Tatlicioglu, and Darren M. Dawson
}

\begin{abstract}
In this paper, control of nonlinear teleoperator systems where both the master and slave systems are kinematically redundant robot manipulators is addressed. The controller is developed under the assumption that the user and environmental input forces are unmeasurable. Lyapunovbased stability analysis is used to prove that the proposed controller yields asymptotic tracking results and ensures the coordination of the master and slave systems while satisfying a sub-task objective.
\end{abstract}

\section{INTRODUCTION}

Teleoperation, where a human operator implements a task in a remote or hazardous environment, has been a mainstream research problem for a long time. Typically, a teleoperator system consists of a local site, where a user operates the master manipulator/system to drive the remotely located slave manipulator/system to implement a given task. Teleoperator systems have significant impact on some common applications including handling of hazardous materials, maneuvering mobile robots, underwater operations, and microsurgery [1], [2], [3], [4]. Transparency [5], keeping the human and the environment safe, proving the boundedness of closed-loop signals, tracking a desired trajectory are important issues in teleoperation. The reader is referred to [6] for a comprehensive overview of research and development in teleoperation over the last several years.

In several robotic applications, including teleoperation, the desired task is usually defined in the Cartesian coordinate frame attached to the robot manipulator's end-effector, which is commonly referred to as the task-space. When a robot manipulator has more degrees of freedom than are required to implement a task in its task-space, it is called a kinematically redundant robot manipulator. The use of kinematically redundant robot manipulators usually complicates the control design; however, they provide greater flexibility to the end user to execute sophisticated or complicated tasks such as obstacle avoidance, grasping, locomotion, etc. [7]. The fact, that the number of joints of a redundant robot manipulator is greater than the dimension of the task-space, results in joint motion in the null-space of the Jacobian matrix that does not

This work is supported in part by a DOE Contract, and a Honda Corporation Grant.

* To whom all correspondence should be addressed.

N. Nath and D. M. Dawson are with the Department of Electrical \& Computer Engineering, Clemson University, Clemson, SC 29634-0915 (email: nnath@clemson.edu; darren.dawson@ces.clemson.edu).

E. Tatlicioglu is with the Department of Electrical \& Electronics Engineering, Izmir Institute of Technology, Urla, Izmir 35430 Turkey (e-mail: enver@envertatlicioglu.com). affect the end-effector motion of the manipulator, generally known as self-motion. A user can deploy the self-motion for achieving a sub-task objective while the task-space controller achieves the primary task for a particular application. As noted in [8], [9], and [10], for redundant robots, given a desired task-space trajectory, an infinite number of inverse kinematic solutions exist that complicates the selection of a reasonable desired joint trajectory, to satisfy the control requirements as well as the sub-task objectives. The reader is referred to [11] for different sub-task objectives and to [8], [12], [13], [14], [15], and the references therein for some of the past research related to the controllers for redundant robot manipulators.

Teleoperation with kinematically redundant robot manipulators is an interesting open research area. Some past work that discuss teleoperation with kinematically redundant robot manipulators can be found in [16] and [17]. Hwang et al. [16] described the performance of a teleoperator system where only the slave system was a redundant robot manipulator. This work failed to provide robustness when the robot operates close to its kinematic singularities or for high joint velocities. In [16], the controller was developed for the primary task and the sub-task objectives were considered as an add-on to the tracking objective; hence, completion of certain sub-task objectives such as joint-limit avoidance was not guaranteed. Das et al. [17] developed kinematic controllers for redundant teleoperator systems to avoid obstacle collision by utilizing a visual display of the slave environment system where just the task-space control was applied. While addressing an interesting problem, [16] and [17] failed to make complete use of the properties of the redundant manipulators.

This work aimed to develop a novel control scheme for teleoperator systems where master and slave systems are kinematically redundant robot manipulators. The control objectives are to track a desired task-space trajectory, to ensure the coordination between the master and the slave systems and to utilize the self-motion of the redundant robot manipulators to achieve sub-task objectives. The control problem is further complicated due to unmeasurable user and environmental input forces. To achieve the control objectives, first the dynamic models of the master and slave systems are put together to form a combined dynamic model for the teleoperator system. Then, robust integral of the sign of the error terms are utilized in the design of a force observer to estimate the unmeasurable input forces. Next, a null-space decomposition is utilized to decompose the control input and the input forces into their task-space and null space 
components; thus, the control development is separated into two parts, namely task-space control and null-space control. To facilitate the task-space control development, an invertible transformation [18] is utilized to encode the control objectives. Another continuous nonlinear observer is designed to estimate the task-space component of the input forces. The task-space controller is developed to meet the taskspace tracking objective and to ensure the coordination of the master and slave systems. In the design of the task-space desired trajectory, an optional strategy of encoding a velocity field assist mechanism [18] is provided to help the user in controlling the slave system. The null-space component of the controller is then designed to meet the null-space velocity tracking objective. Finally, a sub-task controller is developed to make use of the kinematic redundancy of the master and slave systems. Lyapunov-based techniques are utilized in the design of the controllers and the force observers.

\section{Dynamic And Kinematic Models}

The dynamic model for a $2 n$-DOF nonlinear teleoperator system consisting of a revolute $n$-DOF master and a revolute $n$-DOF slave system is described by the following expressions

$$
\begin{aligned}
& M_{1}\left(\theta_{1}\right) \ddot{\theta}_{1}+N_{1}\left(\theta_{1}, \dot{\theta}_{1}\right) \dot{\theta}_{1}=T_{1}+F_{H} \\
& M_{2}\left(\theta_{2}\right) \ddot{\theta}_{2}+N_{2}\left(\theta_{2}, \dot{\theta}_{2}\right) \dot{\theta}_{2}=T_{2}+F_{E}
\end{aligned}
$$

where $\theta_{i}(t), \dot{\theta}_{i}(t), \ddot{\theta}_{i}(t) \in \mathbb{R}^{n}$ denote the joint-space position, velocity, and acceleration for the master and the slave systems, respectively, $M_{i}(\cdot), N_{i}(\cdot) \in \mathbb{R}^{n \times n}$ represent the inertia effects and other dynamic effects, respectively, $T_{i}(t) \in \mathbb{R}^{n}$ represents the control input vector where $i=1$ denotes the master system and $i=2$ denotes the slave system. In (1) and (2), $F_{H}(t) \in \mathbb{R}^{n}$ represents the unmeasurable user input force applied to the master system, and $F_{E}(t) \in \mathbb{R}^{n}$ represents the unmeasurable input force from the environment. The subsequent development utilizes the property that the inertia matrices are positive definite, symmetric and satisfy the following inequalities [19]

$$
m_{1 i}\|\xi\|^{2} \leq \xi^{T} M_{i}(\cdot) \xi \leq m_{2 i}\|\xi\|^{2}
$$

$\forall \xi \in \mathbb{R}^{n}$ and $i=1,2$ where $m_{1 i}, m_{2 i} \in \mathbb{R}$ are positive constants, and $\|\cdot\|$ denotes the standard Euclidean norm.

Assumption 1: To achieve the control objectives, the subsequent development is derived based on the assumption that $\theta_{i}(t), \dot{\theta}_{i}(t)$ are measurable, and $M_{i}(\cdot), N_{i}(\cdot)$ are second order differentiable for $i=1,2$.

Assumption 2: The unmeasurable input forces and their first and second time derivatives, $F_{H}(t), \dot{F}_{H}(t), \ddot{F}_{H}(t)$, $F_{E}(t), \dot{F}_{E}(t)$, and $\ddot{F}_{E}(t)$ are bounded (see [20] and [21] for the precedence of this type of assumption).

The kinematic models for the master and slave systems are described by the following expressions

$$
\begin{aligned}
\dot{x}_{m} & =J_{1}\left(\theta_{1}\right) \dot{\theta}_{1} \\
\dot{x}_{s} & =J_{2}\left(\theta_{2}\right) \dot{\theta}_{2}
\end{aligned}
$$

where $x_{m}(t), x_{s}(t) \in \mathbb{R}^{m}$ are the task-space positions of the master and the slave systems, respectively, and $J_{1}\left(\theta_{1}\right)$,
$J_{2}\left(\theta_{2}\right) \in \mathbb{R}^{m \times n}$ are the Jacobian matrices for the master and the slave systems, respectively. To facilitate the control development, $x(t) \in \mathbb{R}^{2 m}$ and $\theta(t) \in \mathbb{R}^{2 n}$ are defined as follows

$$
\begin{aligned}
x & \triangleq\left[\begin{array}{ll}
x_{m}^{T} & x_{s}^{T}
\end{array}\right]^{T} \\
\theta & \triangleq\left[\begin{array}{ll}
\theta_{1}^{T} & \theta_{2}^{T}
\end{array}\right]^{T} .
\end{aligned}
$$

After utilizing (4)-(7) the following expression can be obtained

$$
\dot{x}=J(\theta) \dot{\theta}
$$

where $J(\cdot) \in \mathbb{R}^{2 m \times 2 n}$ is defined as follows ${ }^{1}$

$$
J \triangleq\left[\begin{array}{cc}
J_{1} & 0_{m \times n} \\
0_{m \times n} & J_{2}
\end{array}\right] .
$$

To facilitate the subsequent development, the dynamic models of the teleoperator system given in (1) and (2) can be combined as follows

$$
M \ddot{\theta}+N \dot{\theta}=T+F
$$

where $M(\cdot), N(\cdot) \in \mathbb{R}^{2 n \times 2 n}, T(\cdot)$, and $F(\cdot) \in \mathbb{R}^{2 n}$ are defined as follows

$$
\begin{aligned}
M & \triangleq\left[\begin{array}{cc}
M_{1} & 0_{n \times n} \\
0_{n \times n} & M_{2}
\end{array}\right] \\
N & \triangleq\left[\begin{array}{cc}
N_{1} & 0_{n \times n} \\
0_{n \times n} & N_{2}
\end{array}\right] \\
T & \triangleq\left[\begin{array}{ll}
T_{1}^{T} & T_{2}^{T}
\end{array}\right]^{T} \\
F & \triangleq\left[\begin{array}{ll}
F_{H}^{T} & F_{E}^{T}
\end{array}\right]^{T} .
\end{aligned}
$$

The kinematic model given in (8) can be written as follows

$$
\dot{x}=J_{f} \theta_{f}
$$

where $\theta_{f}(t) \in \mathbb{R}^{2 n}$ is defined as follows

$$
\theta_{f} \triangleq M \dot{\theta}
$$

and $J_{f}(\theta) \in \mathbb{R}^{2 m \times 2 n}$ is a Jacobian-type matrix, defined as follows

$$
J_{f} \triangleq J M^{-1}
$$

The primary control design objective for teleoperator systems is to ensure the coordination between the master and the slave systems and to meet the tracking objective in the following sense

$$
\begin{aligned}
& x_{s}(t) \rightarrow x_{m}(t) \text { as } t \rightarrow \infty \\
& x_{m}(t) \rightarrow \xi_{1}(t) \text { as } t \rightarrow \infty
\end{aligned}
$$

where $\xi_{1}(t) \in \mathbb{R}^{m}$ is a subsequently designed desired trajectory. Other control objectives are to prove the boundedness of the closed-loop signals and accomplishment of a sub-task objective.

\footnotetext{
${ }^{1}$ Throughout the paper, $0_{i \times j}$ and $I_{i}$ will be used to denote an $i \times j$ zero matrix and an $i \times i$ standard identity matrix, respectively.
} 
Remark 1: The pseudo-inverse of the Jacobian $J_{f}(\theta)$ denoted by $J_{f}^{+}(\theta) \in \mathbb{R}^{2 n \times 2 m}$, is defined as follows

$$
J_{f}^{+} \triangleq J_{f}^{T}\left(J_{f} J_{f}^{T}\right)^{-1}
$$

where $J_{f}^{+}(\theta)$ satisfies the following equality

$$
J_{f} J_{f}^{+}=I_{2 m} \text {. }
$$

As shown in [8], the pseudo-inverse defined by (20) satisfies the Moore-Penrose conditions given below

$$
\begin{array}{rlc}
J_{f} J_{f}^{+} J_{f} & =J_{f} & J_{f}^{+} J_{f} J_{f}^{+}=J_{f}^{+} \\
\left(J_{f}^{+} J_{f}\right)^{T}=J_{f}^{+} J_{f} & \left(J_{f} J_{f}^{+}\right)^{T}=J_{f} J_{f}^{+} .
\end{array}
$$

Additionally, the matrix $\left(I_{2 n}-J_{f}^{+} J_{f}\right)$ satisfies the following properties

$$
\begin{aligned}
\left(I_{2 n}-J_{f}^{+} J_{f}\right)\left(I_{2 n}-J_{f}^{+} J_{f}\right) & =I_{2 n}-J_{f}^{+} J_{f} \\
\left(I_{2 n}-J_{f}^{+} J_{f}\right)^{T} & =I_{2 n}-J_{f}^{+} J_{f} \\
J_{f}\left(I_{2 n}-J_{f}^{+} J_{f}\right) & =0_{2 m \times 2 n} \\
\left(I_{2 n}-J_{f}^{+} J_{f}\right) J_{f}^{+} & =0_{2 n \times 2 m} \\
\left(I_{2 n}-J_{f}^{+} J_{f}\right) J_{\phi} & =J_{\phi}
\end{aligned}
$$

where $J_{\phi}(t) \in \mathbb{R}^{2 n \times 2 n}$ is defined as follows ${ }^{2}$

$$
J_{\phi} \triangleq \dot{J}_{f}^{+} J_{f}+J_{f}^{+} \dot{J}_{f} J_{f}^{+} J_{f} \text {. }
$$

Remark 2: During the control development, it is assumed that the minimum singular value of the manipulator Jacobian matrix, denoted by $\sigma_{m}$, is greater than a known small positive constant $\delta>0$, such that $\max \left\{\left\|J_{f}^{+}(\theta)\right\|\right\}$ is known a priori and all kinematic singularities are always avoided.

\section{FORCE OBSERVER DEVELOPMENT}

In this section, a nonlinear force observer is designed to compensate for the unmeasurable user and environmental forces.

\section{A. Closed-Loop Error System}

Taking the time derivative of (16), results in the following expression

$$
\dot{\theta}_{f}=\dot{M} \dot{\theta}+T+F-N \dot{\theta}
$$

where (10) was utilized. Based on the assumption of the exact model knowledge, the control input is designed as follows

$$
T \triangleq u+(N-\dot{M}) \dot{\theta}
$$

where $u(t) \in \mathbb{R}^{2 n}$ is a subsequently designed auxiliary control input. After substituting (30) into (29), the following simplified expression can be obtained

$$
\dot{\theta}_{f}=u+F \text {. }
$$

The estimate of (31) is defined as follows

$$
\dot{\hat{\theta}}_{f} \triangleq u+\hat{F}
$$

where $\hat{F}(t) \in \mathbb{R}^{2 n}$ is the estimate of $F(t)$ which is to be designed and $\hat{\theta}_{f}(t)$ is the estimate of $\theta_{f}(t)$. To facilitate the

\footnotetext{
${ }^{2}$ The reader is referred to [22] for the proof of (27).
}

development of the closed-loop error system, the observer error, denoted by $\tilde{\theta}_{f}(t) \in \mathbb{R}^{2 n}$, is defined as follows

$$
\tilde{\theta}_{f} \triangleq \hat{\theta}_{f}-\theta_{f}
$$

After taking the time derivative of (33), the following expression can be obtained

$$
\dot{\tilde{\theta}}_{f}=\hat{F}-F
$$

where (31) and (32) were utilized. A filtered observer error signal, denoted by $r_{f}(t) \in \mathbb{R}^{2 n}$, is defined as follows

$$
r_{f} \triangleq \dot{\tilde{\theta}}_{f}+\tilde{\theta}_{f}
$$

The error system dynamics for $r_{f}(t)$ can be derived by taking time derivative of (35)

$$
\dot{r}_{f}=\dot{\hat{F}}-\dot{F}+r_{f}-\tilde{\theta}_{f}
$$

where the time derivatives of (34) and (35) were utilized. Based on the subsequent stability analysis, the proportionalintegral like nonlinear observer $\hat{F}(t)$ is designed as follows

$$
\begin{aligned}
\hat{F} \triangleq & -\left(K_{s}+1\right)\left[\tilde{\theta}_{f}(t)-\tilde{\theta}_{f}\left(t_{0}\right)+\int_{t_{0}}^{t} \tilde{\theta}_{f}(\tau) d \tau\right] \\
& -\left(\beta_{1}+\beta_{2}\right) \int_{t_{0}}^{t} \operatorname{sgn}\left(\tilde{\theta}_{f}(\tau)\right) d \tau
\end{aligned}
$$

where $K_{s}, \beta_{1}, \beta_{2} \in \mathbb{R}$ are positive control gains, and $\operatorname{sgn}(\cdot)$ denotes the vector signum function applied to each element of $\tilde{\theta}_{f}(t)$. In (37), the term $\tilde{\theta}_{f}\left(t_{0}\right)$ is used to ensure that $\hat{F}\left(t_{0}\right)=0_{2 n \times 1}$. The time derivative of (37) is obtained as follows

$$
\dot{\hat{F}}=-\left(K_{s}+1\right) r_{f}-\left(\beta_{1}+\beta_{2}\right) \operatorname{sgn}\left(\tilde{\theta}_{f}\right)
$$

where (35) was utilized. Substituting (38) into (36) results in the following closed-loop error system

$$
\dot{r}_{f}=-K_{s} r_{f}-\left(\beta_{1}+\beta_{2}\right) \operatorname{sgn}\left(\tilde{\theta}_{f}\right)-\dot{F}-\tilde{\theta}_{f} .
$$

Remark 3: After utilizing (14) and Assumption 2, $\|\dot{F}(t)\|$ and $\|\ddot{F}(t)\|$ can be upper bounded as follows

$$
\|\dot{F}(t)\| \leq \varsigma_{1} \quad\|\ddot{F}(t)\| \leq \varsigma_{2}
$$

where $\varsigma_{1}, \varsigma_{2} \in \mathbb{R}$ represent positive bounding constants.

\section{B. Stability Analysis}

Theorem 1: The observer given in (37) guarantees that all signals are bounded and $\hat{F}(t) \rightarrow F(t)$ as $t \rightarrow \infty$ provided the control gain $\beta_{1}$, introduced in (37) is selected to satisfy the following sufficient condition

$$
\beta_{1}>\varsigma_{1}+\varsigma_{2}
$$

where $\varsigma_{1}$ and $\varsigma_{2}$ were introduced in (40).

Proof: See Theorem 5 in [11] for a similar proof. 


\section{TASK-Space Controller Development}

To facilitate the task-space control development, an invertible transformation that encodes the control objectives is defined as follows ${ }^{3}$

$$
\bar{x} \triangleq S x+\left[\begin{array}{c}
0_{m \times 1} \\
\xi_{2}
\end{array}\right]
$$

where $\bar{x} \in \mathbb{R}^{2 m}, \xi_{2}(t) \in \mathbb{R}^{m}$ is an auxiliary signal that will be designed subsequently and $S \in \mathbb{R}^{2 m \times 2 m}$ is defined as follows

$$
S \triangleq\left[\begin{array}{ll}
I_{m} & 0_{m \times m} \\
I_{m} & -I_{m}
\end{array}\right] .
$$

To meet the control objectives, a filtered tracking error signal, denoted by $r_{T}(t) \in \mathbb{R}^{2 m}$, is defined as follows

$$
r_{T} \triangleq \dot{s}_{T}+s_{T}
$$

where $s_{T}(t) \in \mathbb{R}^{2 m}$ is defined as follows

$$
s_{T} \triangleq \dot{e}_{T}+e_{T}
$$

where $e_{T}(t) \in \mathbb{R}^{2 m}$ is defined as follows

$$
e_{T} \triangleq \xi_{d}-\bar{x} \text {. }
$$

where $\xi_{d}(t) \in \mathbb{R}^{2 m}$ is defined as follows

$$
\xi_{d} \triangleq\left[\begin{array}{ll}
\xi_{1}^{T} & \xi_{2}^{T}
\end{array}\right]^{T}
$$

where $\xi_{1}(t) \in \mathbb{R}^{m}$ is the actual desired trajectory that will be tracked by the master and the slave systems. After utilizing (6), (42), and (47), the error signal $e_{T}(t)$ can be written as follows

$$
e_{T} \triangleq\left[\begin{array}{l}
e_{1} \\
e_{2}
\end{array}\right]=\left[\begin{array}{l}
\xi_{1}-x_{m} \\
x_{s}-x_{m}
\end{array}\right]
$$

where $e_{1}(t) \in \mathbb{R}^{m}$ represents the tracking error between the master system and the desired trajectory $\xi_{1}(t)$, and $e_{2}(t) \in$ $\mathbb{R}^{m}$ represents the coordination error between the master and the slave systems. From (48), it is clear that if $\left\|e_{T}(t)\right\| \rightarrow 0$ then $x_{s}(t) \rightarrow x_{m}(t)$ and $x_{m}(t) \rightarrow \xi_{1}(t)$.

Remark 4: The input force defined in (14) can be decomposed as follows

$$
F \triangleq J_{f}^{+} F_{T}+\left(I_{2 n}-J_{f}^{+} J_{f}\right) F_{N}
$$

where $F_{T}(t) \in \mathbb{R}^{2 m}$ and $F_{N}(t) \in \mathbb{R}^{2 n}$ are the taskspace and the null-space components of the unmeasurable input forces. The auxiliary control input, $u(t)$ is designed as follows

$$
u \triangleq J_{f}^{+} u_{T}+\left(I_{2 n}-J_{f}^{+} J_{f}\right) u_{N}
$$

where $u_{T}(t) \in \mathbb{R}^{2 m}$ and $u_{N}(t) \in \mathbb{R}^{2 n}$ represent subsequently designed task-space and null-space components of the auxiliary control input, respectively.

To compensate for the task-space component of the unmeasurable input forces, a nonlinear force observer is designed subsequently which is also utilized to drive the target

\footnotetext{
${ }^{3}$ The reader is referred to [18] for a detailed explanation of the transformation.
}

system. The desired trajectory $\xi_{d}(t)$ is generated by the following second order coupled dynamic target system [18]

$$
\begin{gathered}
\dot{\xi}_{d}=\gamma\left[\begin{array}{l}
\varphi\left(\xi_{1}\right) \\
0_{m \times 1}
\end{array}\right]+\eta_{d} \\
\dot{\eta}_{d}+B_{T} \eta_{d}+C_{T} \lambda_{d}=\hat{F}_{T}
\end{gathered}
$$

where $\eta_{d}(t) \in \mathbb{R}^{2 m}$ is an auxiliary filter signal, $B_{T}$, $C_{T} \in \mathbb{R}^{2 m \times 2 m}$ represent constant, positive definite, diagonal matrices, $\hat{F}_{T}(t) \in \mathbb{R}^{2 m}$ is a subsequently designed nonlinear observer to estimate $F_{T}(t), \varphi(\cdot) \in \mathbb{R}^{m}$ is a velocity field function [23] that encodes the user assist mechanism, and $\gamma$ is a constant gain that is either 0 or 1 . It should be noted that, when $\gamma=1$, the user assist mechanism is enabled and it is disabled when $\gamma=0$. Also, in (52) the term $\lambda_{d}(t) \in \mathbb{R}^{2 m}$ is defined as follows

$$
\lambda_{d} \triangleq \xi_{d}-\gamma\left[\begin{array}{c}
\int_{t_{0}}^{t} \varphi\left(\xi_{1}(\tau)\right) d \tau \\
0_{m \times 1}
\end{array}\right] .
$$

Remark 5: The velocity field function $\varphi(\cdot)$ is assumed to be designed such that, from (51), if $\eta_{d}(t) \in \mathcal{L}_{\infty}$ then $\xi_{d}(t), \dot{\xi}_{d}(t) \in \mathcal{L}_{\infty}$. Subsequent analysis shows that $\hat{F}_{T}(t) \in$ $\mathcal{L}_{\infty}$. These facts can be utilized along with the analysis in Appendix $G$ of [11] to prove that all the signals in the dynamic target system along with their higher order derivatives are bounded.

\section{A. Closed-Loop Error System}

Taking the time derivative of (15) results in the following expression

$$
\ddot{x}=u_{T}+F_{T}+\dot{J}_{f} \theta_{f}
$$

where (21), (25), (31), (49), and (50) were utilized. After taking the second time derivative of (42), the following expression is obtained

$$
\ddot{\bar{x}}=S\left(u_{T}+\dot{J}_{f} \theta_{f}\right)+\bar{F}_{T}+\left[\begin{array}{c}
0_{m \times 1} \\
\ddot{\xi}_{2}
\end{array}\right]
$$

where (54) was utilized and $\bar{F}_{T}(t) \in \mathbb{R}^{2 m}$ is defined as follows

$$
\bar{F}_{T} \triangleq S F_{T} .
$$

To facilitate the closed-loop error system for $r_{T}(t)$, the error system dynamics for $s_{T}(t)$ and $e_{T}(t)$ are derived first. After taking the second order derivative of (46), the following expression is obtained

$$
\begin{aligned}
\ddot{e}_{T}= & \gamma\left[\begin{array}{c}
\dot{\varphi}\left(\xi_{1}\right) \\
0_{m \times 1}
\end{array}\right]+\hat{F}_{T}-B_{T} \eta_{d}-C_{T} \lambda_{d} \\
& -S\left(u_{T}+\dot{J}_{f} \theta_{f}\right)-\bar{F}_{T}-\left[\begin{array}{c}
0_{m \times 1} \\
\ddot{\xi}_{2}
\end{array}\right]
\end{aligned}
$$

where (51), (52) and (55) were utilized. The task-space component of the auxiliary control input, $u_{T}(t)$ is designed as follows

$$
\begin{aligned}
u_{T} \triangleq & -\dot{J}_{f} \theta_{f}+S^{-1}\left(\gamma\left[\begin{array}{l}
\dot{\varphi}\left(\xi_{1}\right) \\
0_{m \times 1}
\end{array}\right]-B_{T} \eta_{d}\right. \\
& \left.-C_{T} \lambda_{d}-\left[\begin{array}{c}
0_{m \times 1} \\
\ddot{\xi}_{2}
\end{array}\right]\right)+S^{-1} \bar{u}_{T}
\end{aligned}
$$


where $\bar{u}_{T}(t) \in \mathbb{R}^{2 m}$ is a subsequently designed auxiliary control input. After substituting (58) into (57), the following simplified expression is obtained

$$
\ddot{e}_{T}=\hat{F}_{T}-\bar{F}_{T}-\bar{u}_{T} \text {. }
$$

The time derivative of $s_{T}(t)$ can be obtained as follows

$$
\dot{s}_{T}=\hat{F}_{T}-\bar{F}_{T}-\bar{u}_{T}+s_{T}-e_{T}
$$

where (45) and (59) were utilized. Based on (60), the auxiliary control input $\bar{u}_{T}(t)$ is designed as follows

$$
\bar{u}_{T} \triangleq s_{T}-e_{T} \text {. }
$$

After substituting (61) into (60), the following simplified expression is obtained

$$
\dot{s}_{T}=\hat{F}_{T}-\bar{F}_{T} .
$$

The error system dynamics for $r_{T}(t)$ can be derived by taking the time derivative of (44)

$$
\dot{r}_{T}=\dot{\hat{F}}_{T}-\dot{\bar{F}}_{T}+r_{T}-s_{T}
$$

where (44) and the time derivative of (62) were utilized. Based on (63), the proportional-integral like nonlinear observer introduced in (52) is designed as follows

$$
\begin{aligned}
\hat{F}_{T} \triangleq & -\left(K_{T}+1\right)\left[s_{T}(t)-s_{T}\left(t_{0}\right)+\int_{t_{0}}^{t} s_{T}(\tau) d \tau\right] \\
& -\left(\beta_{3}+\beta_{4}\right) \int_{t_{0}}^{t} \operatorname{sgn}\left(s_{T}(\tau)\right) d \tau
\end{aligned}
$$

where $K_{T}, \beta_{3}, \beta_{4} \in \mathbb{R}$ are positive control gains. In (64), the term $s_{T}\left(t_{0}\right)$ ensures that $\hat{F}_{T}\left(t_{0}\right)=0_{2 m \times 1}$. The time derivative of (64) is obtained as follows

$$
\dot{\hat{F}}_{T}=-\left(K_{T}+1\right) r_{T}-\left(\beta_{3}+\beta_{4}\right) \operatorname{sgn}\left(s_{T}\right)
$$

where (44) was utilized. After substituting (65) into (63), the following closed-loop error system is obtained

$$
\dot{r}_{T}=-K_{T} r_{T}-\left(\beta_{3}+\beta_{4}\right) \operatorname{sgn}\left(s_{T}\right)-\dot{\bar{F}}_{T}-s_{T} .
$$

Remark 6: After utilizing (14), Assumption 2, (49), and (56), $\left\|\dot{\bar{F}}_{T}(t)\right\|$ and $\left\|\ddot{\bar{F}}_{T}(t)\right\|$ can be upper bounded as follows

$$
\left\|\dot{\bar{F}}_{T}(t)\right\| \leq \varsigma_{3} \quad\left\|\ddot{\bar{F}}_{T}(t)\right\| \leq \varsigma_{4}
$$

where $\varsigma_{3}, \varsigma_{4} \in \mathbb{R}$ represent positive bounding constants.

\section{B. Stability Analysis}

Theorem 2: The controller given in (58) and (61) and the observer in (64) guarantee that all signals are bounded under the closed-loop operation and that coordination between the master and slave systems, and the tracking objective are met in the sense that

$$
\begin{aligned}
& x_{s}(t) \rightarrow x_{m}(t) \text { as } t \rightarrow \infty \\
& x_{m}(t) \rightarrow \xi_{1}(t) \text { as } t \rightarrow \infty
\end{aligned}
$$

provided the control gain $\beta_{3}$, introduced in (64) is selected to satisfy the sufficient condition

$$
\beta_{3}>\varsigma_{3}+\varsigma_{4} \text {. }
$$

Proof: See Theorem 5 in [11] for a similar proof.

\section{Null-Space Controller Development}

In this section the null-space controller $u_{N}(t)$ will be designed to meet the null-space velocity tracking objective. In the controller design, the redundancy of the master and the slave manipulators will be utilized to perform certain sub-task objectives required for a particular application. To integrate this sub-task objective into the controller, an auxiliary control signal, denoted by $g(t)$, will be introduced. This auxiliary controller is designed through the joint motion in the null-space of the Jacobian matrix.

The null-space velocity tracking error, denoted by $\dot{e}_{N}(t) \in$ $\mathbb{R}^{2 n}$, is defined as [24]

$$
\dot{e}_{N} \triangleq\left(I_{2 n}-J_{f}^{+} J_{f}\right)\left(g-\theta_{f}\right)
$$

where $g(t) \in \mathbb{R}^{2 n}$ is the subsequently designed sub-task controller. After utilizing (31), (49), and (50) the following expression is obtained

$$
\dot{\theta}_{f}=J_{f}^{+} u_{T}+J_{f}^{+} F_{T}+\left(I_{2 n}-J_{f}^{+} J_{f}\right)\left(u_{N}+F_{N}\right) .
$$

After taking the time derivative of (71), the dynamics of $\dot{e}_{N}(t)$ can be obtained as follows

$$
\begin{aligned}
\ddot{e}_{N}= & \left(I_{2 n}-J_{f}^{+} J_{f}\right) \dot{g}-\left(\dot{J}_{f}^{+} J_{f}+J_{f}^{+} \dot{J}_{f}\right)\left(g-\theta_{f}\right) \\
& -\left(I_{2 n}-J_{f}^{+} J_{f}\right)\left(u_{N}+F_{N}\right)
\end{aligned}
$$

where (21), (23), (26), (71) and (72) were utilized. After adding and subtracting the term $J_{f}^{+} \dot{J}_{f} J_{f}^{+} J_{f}\left(g-\theta_{f}\right)$ to the right-hand-side of (73), the following expression is obtained

$$
\begin{aligned}
\ddot{e}_{N}= & \left(I_{2 n}-J_{f}^{+} J_{f}\right)\left(\dot{g}-u_{N}\right)+J_{f}^{+} F_{T}-F \\
& -J_{\phi}\left(g-\theta_{f}\right)-J_{f}^{+} \dot{J}_{f} \dot{e}_{N}
\end{aligned}
$$

where (28), (49), and (71) were utilized. The null-space component of the auxiliary control input $u_{N}(t)$, introduced in (50), is now designed as follows

$$
u_{N} \triangleq-J_{\phi}\left(g-\theta_{f}\right)+\dot{g}+K_{N} \dot{e}_{N}-\hat{F}
$$

where $K_{N} \in \mathbb{R}$ is a positive constant. After substituting (75) into (74) and utilizing (21)-(27) the following expression for the dynamics of $\dot{e}_{N}(t)$ is obtained

$$
\begin{aligned}
\ddot{e}_{N}= & -\left(I_{2 n}-J_{f}^{+} J_{f}\right)\left(K_{N} \dot{e}_{N}-\hat{F}\right) \\
& +J_{f}^{+} F_{T}-F-J_{f}^{+} \dot{J}_{f} \dot{e}_{N} .
\end{aligned}
$$

Theorem 3: The null-space component of the control input described by (75) guarantees that the null-space velocity tracking is obtained in the sense that $\left\|\dot{e}_{N}(t)\right\| \rightarrow 0$ as $t \rightarrow \infty$.

Proof: See [22].

\section{Sub-Task Controller Development}

In this section, the sub-task controller $g(t)$ is developed. The subsequent stability analysis shows that the sub-task objective is met if the Jacobian-related null space matrix maintains full-rank.

An auxiliary positive function $y_{a i}(t) \in \mathbb{R}$ is defined as follows

$$
y_{a i} \triangleq \exp \left(-k_{i} \rho_{i}\left(\theta_{i}\right)\right) \quad \forall i=1,2
$$


where $i=1$ and 2 for the master and the slave systems, respectively and $k_{i} \in \mathbb{R}$ is a positive constant, $\rho_{i}(\cdot) \in \mathbb{R}$ is a nonnegative function that is specific to a sub-task, and $\exp (\cdot)$ is the natural logarithmic exponential function. To facilitate the sub-task controller development, a positive function $y_{a}(t) \in \mathbb{R}$ is defined as follows

$$
y_{a} \triangleq y_{a 1}+y_{a 2}
$$

After taking the time derivative of (78), the following expression can be obtained

$$
\dot{y}_{a}=J_{s} \dot{\theta}
$$

where (7) was utilized and $J_{s}(t) \in \mathbb{R}^{1 \times 2 n}$ is a Jacobian-type vector defined as follows

$$
J_{s} \triangleq\left[\begin{array}{ll}
\frac{\partial y_{a 1}}{\partial \theta_{1}} & \frac{\partial y_{a 2}}{\partial \theta_{2}}
\end{array}\right]
$$

After utilizing (16), (79) can be expressed as follows

$$
\dot{y}_{a}=\bar{J}_{s} \theta_{f}
$$

where the term $\bar{J}_{s} \triangleq J_{s} M^{-1} \in \mathbb{R}^{1 \times 2 n}$ is again a Jacobiantype vector. After adding and subtracting the term $\bar{J}_{s}\left(I_{2 n}-\right.$ $\left.J_{f}^{+} J_{f}\right)\left(g-\theta_{f}\right)$ to the right-hand-side of (81) the following simplified expression is obtained

$$
\dot{y}_{a}=\bar{J}_{s}\left(I_{2 n}-J_{f}^{+} J_{f}\right) g-\bar{J}_{s} J_{f}^{+} J_{f} \theta_{f}-\bar{J}_{s} \dot{e}_{N}
$$

where (71) was utilized. Based on the subsequent stability analysis, the sub-task controller is designed as follows

$$
g \triangleq-K_{g} \bar{J}_{s}^{T} y_{a}
$$

where $K_{g} \in \mathbb{R}$ is a positive constant. After substituting (83) into (82), the following expression is obtained

$$
\dot{y}_{a}=-K_{g} \bar{J}_{s}\left(I_{2 n}-J_{f}^{+} J_{f}\right) \bar{J}_{s}^{T} y_{a}-\bar{J}_{s} J_{f}^{+} J_{f} \theta_{f}-\bar{J}_{s} \dot{e}_{N} .
$$

Theorem 4: The control law described by (83) guarantees that $y_{a}(t)$ is ultimately bounded in the following sense

$$
\left|y_{a}(t)\right| \leq \sqrt{\left|y_{a}^{2}\left(t_{0}\right)\right| \exp (-2 \nu t)+\frac{\varepsilon}{\nu}}
$$

provided that

$$
K_{g}>\frac{1}{\bar{\delta}}
$$

where $\varepsilon, \nu, \bar{\delta} \in \mathbb{R}$ are positive constants.

Proof: See Theorem 2 in [11] for a similar proof.

\section{CONCLUSION}

A controller for nonlinear teleoperator systems where both the master and slave systems are kinematically redundant robot manipulators was presented. Lyapunov-based stability analysis was used to show that the proposed controller yields asymptotic results despite the unmeasurable user and environmental input forces. Also, the kinematic redundancy of the teleoperator system was utilized to integrate a subtask controller. The proposed controller ensured that the master system accurately tracks a desired task-space trajectory, coordinates the master and the slave systems and additionally, satisfies sub-task objectives. An optional user assist mechanism was also presented that assists the user of the system to complete a pre-defined contour with the slave system.

\section{REFERENCES}

[1] T. B. Sheridan, "Teleoperation, telerobotics and telepresence: A progress report," Control Engineering Practice, vol. 3, no. 2, pp. 205214, 1995.

[2] P. Berkelman and J. Ma, "A compact, modular, teleoperated minimally invasive surgery system," in Proc. IEEE/RAS-EMBS Int. Conf. Biomed. Robotics and Biomechatronics, Pisa, Italy, Feb. 2006, pp. 702-707.

[3] F. Ogaki and K. Suzuki, "Adaptive teleoperation of a mobile robot under communication time delay," in IEEE Int. Workshop on ROSE, Ottawa, Canada, Oct. 2007, pp. 1-6.

[4] E. Slawinski, J. Postigo, and V. Mutt, "Stable teleoperation of mobile robots," in Proc. IEEE Int. Conf. Mechatronics. Autom., Luoyang, China, Jun 2006, pp. 318-323.

[5] D. A. Lawrence, "Stability and transparency in bilateral teleoperation," IEEE Trans. Robot. Automat., vol. 9, no. 5, pp. 624-637, 1993.

[6] P. F. Hokayem and M. W. Spong, "Bilateral teleoperation: An historical survey," Automatica, vol. 42, no. 12, pp. 2035-2057, 2006.

[7] G. S. Chirikjian and J. W. Burdick, "Hyper-redundant robot mechanisms and their applications," in Proc. IEEE/RSJ Int. Conf. Intell. Robots Syst., Osaka, Japan, Nov 1991, pp. 185-190.

[8] Y. Nakamura, Advanced Robotics Redundancy and Optimization. Reading, MA: Addison-Wesley, 1991.

[9] D. N. Nenchev, "Redundancy resolution through local optimization: A reveiw," J. Robotic Systems, vol. 6, no. 6, pp. 769-798, 1993.

[10] B. Siciliano, "Kinematic control of redundant robot manipulators: A tutorial," J. Intell. Robotic Systems, vol. 3, pp. 201-212, 1990.

[11] E. Tatlicioglu, Control of Nonlinear Mechatronic Systems. Germany: VDM Verlag Dr. Mueller e.K., 2008.

[12] W. E. Dixon, A. Behal, D. M. Dawson, and S. Nagarkatti, Nonlinear Control of Engineering Systems: A Lyapunov-Based Approach. Boston, MA: Birkhauser, 2003.

[13] E. Zergeroglu, D. Dawson, I. Walker, and A. Behal, "Nonlinear tracking control of kinematically redundant robot manipulators," in Proc. American Control Conf., Chicago, IL, Jun. 2000, pp. 2513-2517.

[14] R. Colbaugh and K. Glass, "Robust adaptive control of redundant manipulators," J. Intell. Robotic Systems, vol. 14, no. 1, pp. 68-88, 1995.

[15] J. Yuan, "A general photogrammetric method for determining object position and orientation," IEEE Trans. Robot. Autom., vol. 5, no. 2, pp. 129-142, 1989.

[16] D. Hwang and B. Hannaford, "Teleoperation performance with a kinematically redundant slave robot," Int. J. Robotics Research, vol. 17, pp. 579-597, 1998.

[17] H. Das, T. B. Sheridan, and J. J. E. Slotine, "Kinematic control and visual display of redundant teleoperators," in IEEE Int. Conf. Syst., Man, Cybern., Cambridge, MA, Nov. 1989, pp. 1072-1077.

[18] E. Tatlicioglu, M. McIntyre, D. M. Dawson, and T. Burg, "Coordination control for haptic and teleoperator systems," in Proc. IEEE Int. Conf. Decision and Control, San Diego, CA, Dec. 2006, pp. 29372942.

[19] F. Lewis, D. Dawson, and C. Abdallah, Robot Manipulator Control: Theory and Practice. New York, NY: Marcel Dekker, Inc., 2004.

[20] D. Lee and P. Y. Li, "Passive coordination of nonlinear bilateral teleoperated manipulators," in Proc. IEEE Int. Conf. Robot. Autom., Washington DC, May. 2002, pp. 3278-3283.

[21] _ , "Passive bilateral feedforward control of linear dynamically similar teleoperated manipulators," IEEE Trans. Robot. Autom., vol. 19, no. 3, pp. 443-456, 2003.

[22] N. Nath, E. Tatlicioglu, and D. M. Dawson, "Teleoperation with kinematically redundant robot manipulators with sub-task objectives," Clemson University CRB, Tech. Rep. CU/CRB/2/28/08/1, Feb. 2008. [Online]. Available: http://www.ces.clemson.edu/ece/crb/publictn/tr.htm

[23] P. Li and R. Horowitz, "Passive velocity field control of mechanical manipulators," IEEE Trans. Robot. Automat., vol. 15, no. 4, pp. 751763, 1999.

[24] P. Hsu, J. Hauser, and S. Sastry, "Dynamic control of redundant robot manipulators," Journal of Robotic Systems, vol. 6, no. 3, pp. 133-148, 1989. 